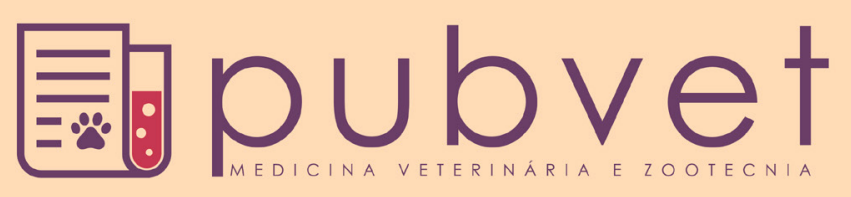

HTTP://DX.DOI.ORG/10.22256/PUBVET.V11N8.767-770

\title{
Anatomia macroscópica dos órgãos reprodutores do Puma yagouaroundi (Geoffroy, 1803) macho
}

\author{
Ediane Freitas Rocha ${ }^{1}$, Nayadjala Távita Alves dos Santos ${ }^{1}$, Rômulo Freitas Francelino \\ Dias $^{1}$, João Augusto Rodrigues Alves Diniz ${ }^{1}$, José Rômulo Soares dos Santos ${ }^{1}$, Danilo José \\ Ayres de Menezes ${ }^{2 *}$ \\ ${ }^{1}$ Universidade Federal de Campina Grande - Patos, $P B$ \\ ${ }^{2}$ Universidade Federal do Rio Grande do Norte - Natal, $R N$ \\ *Autor para correspondência: UFRN/CB/DMOR, Campus Universitário Lagoa Nova, Cx. Postal 1524 CEP - 59078-970. \\ Natal, Rio Grande do Norte, Brasil.Tel.: +55835113000.Endereço de e-mail: mdanayres@gmail.com
}

RESUMO. O Puma yagouaroundi (gato-mourisco) é um felino que apresenta vasta distribuição geográfica no Brasil, podendo ser encontrado em vários biomas. Na literatura observa-se escassez de trabalhos sobre sua anatomia macroscópica. O objetivo do trabalho foi a descrição macroscópica do sistema reprodutor masculino visando a obtenção de informações que possam ser úteis ao manejo reprodutivo da espécie. O estudo foi realizado em um cadáver macho doado ao Laboratório de Anatomia Veterinária pelo Hospital Veterinário da Universidade Federal de Campina Grande. O animal foi fixado em formal a $10 \%$ para posterior dissecação e descrição do sistema reprodutor masculino. Foram identificadas as seguintes estruturas funcionais do aparelho genital masculino do gatomourisco: escroto, pênis, testículos, epidídimos, ductos deferentes e as glândulas genitais acessórias, identificando-se apenas a próstata, não possuindo as glândulas bulbouretrais e vesiculares. $\mathrm{O}$ escroto com seus constituintes internos situa-se na região subanal. Os testículos apresentaram formato ovóide, posicionado perinealmente, dentro do escroto. $\mathrm{O}$ ducto deferente compreende uma estrutura longa, contínua a cauda do epidídimo, desembocando na superfície craniodorsal da próstata. O pênis mostrou-se um órgão curto, achatado látero-lateralmente com presença de espículas e localizado dentro do prepúcio.

Palavras Chaves: descrição macroscópica, gato mourisco, sistema reprodutor

\section{Gross anatomy of the reproductive organs of male Puma yagouaroundi (Geoffroy, 1803)}

ABSTRACT: The Puma yagouaroundi (cat-Moorish) is a feline that has wide geographic distribution in Brazil, can be found in various biomes. In the literature there has been few studies on their gross anatomy. The objective was the macroscopic description of the male reproductive system in order to obtain information that might be useful to reproductive management of the species. The study was conducted in a male body donated to Veterinary Anatomy Laboratory at the Veterinary Hospital of the Federal University of Campina Grande. The animal was fixed in 10\% formal for further dissection and description of the male reproductive system. the following functional structures of the male genital tract of the cat-Moorish were identified: scrotum, penis, testicles, epididymis, vas deferens and accessory genital glands, identifying only the prostate, not having the bulbourethral and vesicular glands. The scrotum, with its internal components is located in subanal region. The testes showed ovoid shape, positioned perinealmente within the scrotum. The vas deferens comprises a long structure, continuous tail of the epididymis, ending up in 
craniodorsal surface of the prostate. The penis proved to be a short body, flattened side-toside with the presence of spicules and located within the prepuce.

Keywords: Macroscopic description, moorish cat, reproductive system

\section{Anatomía macroscópica de los órganos reproductores del Puma yagouaroundi (Geoffroy, 1803) macho}

RESUMEN. El Puma yagouaroundi (yaguarundí, zorro-gato) es un felino que presenta una amplia distribución geográfica en Brasil, pudiendo ser encontrado en varios biomas. En la literatura se observa escasez de trabajos sobre su anatomía macroscópica. El objetivo del trabajo fue la descripción macroscópica del sistema reproductivo masculino, buscando obtener informaciones que puedan ser útiles al manejo reproductivo de la especie. El estudio fue realizado en un cadáver macho donado por el Hospital Veterinario de la Universidad Federal de Campina Grande. El animal fue fijado en formol al 10\% para posterior disección y descripción del sistema reproductor masculino. Se identificaron las siguientes estructuras funcionales del aparato genital masculino del gato-morisco: escroto, pene, testículos, epidídimos, conductos deferentes y las glándulas genitales accesorias, identificándose sólo la próstata, no poseyendo las glándulas bulbouretrales y vesiculares. El escroto con sus constituyentes internos se sitúa en la región sub anal. Los testículos presentaron forma ovoide, posicionado perinealmente, dentro del escroto. El conducto deferente comprende una estructura larga, continua la cola del epidídimo, desembocando en la superficie craneodorsal de la próstata. El pene se mostró un órgano corto, achatado latero-lateralmente con presencia de espículas y localizado dentro del prepucio.

Palabras claves: descripción macroscópica, gato morisco, sistema reproductor

\section{Introdução}

O Gato-mourisco (Puma yagouaroundi Geoffroy, 1803), também conhecido como jaguarundi, gato vermelho, gato colorado, entre outros, pertence à ordem Carnívora e família Felidae. Apresenta vasta distribuição geográfica no Brasil, podendo ser encontrado em vários biomas, incluindo florestas tropicais e subtropicais, cerrado, caatinga, pantanal. O Puma yagouaroundi possui hábitos diurnos e noturnos, com atividade predominantemente diurna. Seu peso médio é de $5,2 \mathrm{~kg}$, sendo uma espécie de pequeno porte (Moro-Rios et al., 2008, Almeida et al., 2013). O padrão de pelagem da espécie varia do cinza escuro ao laranja (Maffei et al., 2007). Essa espécie, no Brasil, apesar de não se encontrar na lista dos animais ameaçados de extinção (Cubas et al., 2014) possui uma população efetiva de apenas 5.200 indivíduos e certamente inferior a 10.000 indivíduos, com estimativa de que nos próximos 15 anos (três gerações) poderia ocorrer um declínio de pelo menos $10 \%$ desta população em razão principalmente da perda e fragmentação de habitat pela expansão agrícola. Devido a isso, a espécie é considerada vulnerável (VU) no estado do Rio Grande do Sul (Marques et al., 2002) e como menos preocupante (LC) em São Paulo (Bressan et al., 2009).
O estudo dos aspectos reprodutivos de uma espécie e o desenvolvimento de métodos alternativos de manejo para a manutenção da diversidade genética pode fornecer dados para auxiliar na sua conservação. Particularmente em animais silvestres, onde são escassos os dados na literatura sobre os aspectos reprodutivos, trabalhos sobre a anatomia dos órgãos reprodutores são importantes para subsidiar estudos mais avançados em biotecnologia da reprodução, no desenvolvimento de técnicas para melhorar o desempenho reprodutivo de espécies em cativeiro. Aumentando o sucesso reprodutivo em cativeiro, além de aumentar o número de indivíduos de criadouros, animais podem ser reintroduzidos em reservas, aumentando também a população da espécie, particularmente de animais ameaçados ou em risco de extinção.

Já foram desenvolvidos trabalhos sobre o Puma yagouaroundi (Baptista et al., 2016, Almeida et al., 2013), principalmente sobre comportamento e conservação, porém são poucos os estudos referentes a anatomia macroscópica. Dessa forma, o estudo teve como finalidade obter bases anatômicas macroscópica do sistema reprodutor masculino de Gato Mourisco ( $P$. yagouaroundi) visando à obtenção de informações 
que possam ser úteis ao manejo reprodutivo da espécie.

\section{Material e métodos}

O estudo foi realizado em um espécime de gato-mourisco (Puma yagouaroundi) macho, que deu entrada no Hospital Veterinário da Universidade Federal de Campina Grande após óbito por atropelamento. $\mathrm{O}$ mesmo foi encaminhado ao Laboratório de Anatomia Veterinária. $\mathrm{O}$ animal foi fixado utilizando-se solução aquosa de formol $10 \%$ por meio de perfusão intramuscular e subcutânea. Após a fixação, o animal foi submerso na mesma solução de formol a $10 \%$ para posterior dissecação. A dissecação do sistema reprodutor masculino iniciou-se com a identificação da artéria testicular direita e esquerda. Percorrendo o seu trajeto foi possível identificar o anel inguinal e o ducto deferente. A região perineal, os testículos e o pênis foram dissecados e posteriormente o púbis foi removido para que fosse possível a remoção completa do sistema reprodutor. A nomenclatura adotada foi baseada na Nomina Anatomica Veterinaria (International Committee on Veterinary Gross Anatomical Nomenclature, 2005).

\section{Resultados e discussão}

Foram identificadas as seguintes estruturas funcionais do aparelho genital masculino do gato mourisco: escroto, pênis, testículos, epidídimos, ductos deferentes e as glândulas genitais acessórias, identificando-se apenas a próstata, não sendo identificadas as glândulas bulbouretrais e vesiculares (Figura 1). Os constituintes do aparelho reprodutor masculino do gato mourisco condizem com os descritos por Dyce et al. (2004) para o gato doméstico, exceto por não ter sido identificado as glândulas bulbouretrais na espécie estudada.

$\mathrm{O}$ escroto no $P$. yagouaroundi situa-se na região subanal, seus constituintes incluem internamente a túnica vaginal, dividida em lâmina visceral e lâmina parietal, que recobre o testículo, epidídimo e parte do funículo espermático, a fáscia espermática interna, túnica dartos, camada fibromuscular que divide o escroto em dois compartimentos e que se encontra firmemente aderida a camada mais externa do escroto, a pele.

Os testículos apresentaram formato ovoide, são pequenos, posicionado perinealmente, dentro do escroto, envoltos primeiramente, pela túnica vaginal (lâminas visceral e parietal).

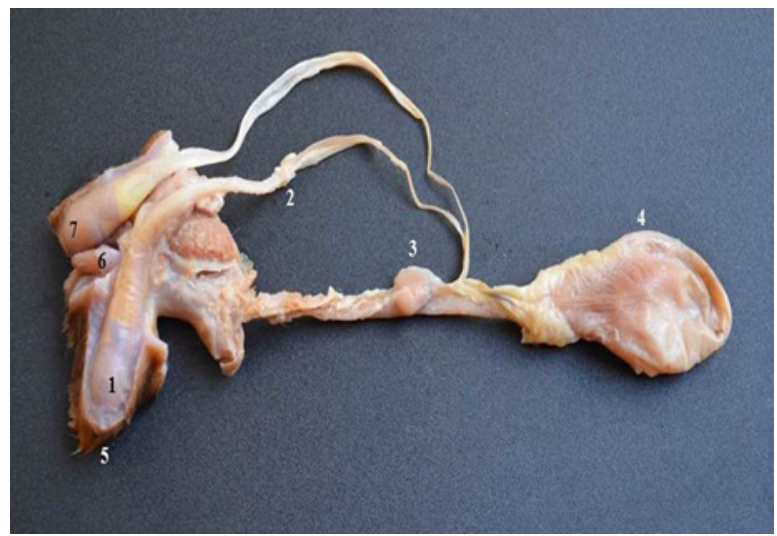

Figura 1 - Fotografia do aparelho genital masculino do gato-mourisco (Puma yagouaroundi). Observam-se as seguintes estruturas: testículo direito (1), ducto deferente (2), próstata (3), vesícula urinária (4), escroto (5), pênis (6) e testículo esquerdo (7).

O epidídimo apresentou-se firmemente aderido ao testículo, não sendo observado o seio epididimário completo, havendo separação apenas na extremidade da cauda do testículo, não sendo possível delimitar a divisão entre cabeça e corpo. $\mathrm{O}$ ducto deferente compreende uma estrutura longa, contínua a cauda do epidídimo, sendo um dos constituintes do funículo espermático até o anel inguinal. Pelo canal inguinal penetra na cavidade abdominal, descrevendo uma trajetória convexa, ligado à parede abdominal com auxílio do mesoducto deferente, passando ventromedial ao ureter e desembocando na superfície craniodorsal da próstata. A única glândula genital acessória identificada no $P$. yagouaroundi foi a próstata, localizada entre uretra pélvica e uretra peniana, apresentando tamanho semelhante a uma ervilha.

O pênis mostrou-se um órgão curto, achatado látero-lateralmente com presença de espículas e localizado dentro do prepúcio. A presença de espículas identificadas na superfície do pênis do $P$. yagouaroundi, difere dos achados de Carneiro et al. (2010) que não observaram tal estrutura ao o estudar a espécie de felino jaguatirica (Leopardus pardalis). Todavia, deve-se considerar que no trabalho realizado por Queiroz (2003); o autor afirma ter identificado espículas no pênis da jaguatirica, embora em alguns animais fossem pouco desenvolvidas. A literatura cita várias funções para as espículas penianas, no entanto, a hipótese mais aceitas quanto a função das espículas é o estímulo causado no trato reprodutor feminino, o que acelera a copulação completa e 
realça a peristalse, movimentando o esperma pelo trato reprodutor feminino (Harcourt and Gardiner, 1994). Conforme mencionado anteriormente, há poucos relatos na literatura sobre a macroscopia do sistema reprodutor masculinos de felinos silvestres sendo descritos apenas trabalhos sobre as características do sêmen (Jayaprakash et al., 2001) e a ocorrência de criptorquidismo (Mansfield and Land, 2002), não sendo citados os aspectos macroscópicos. Isso se deve provavelmente ao difícil acesso aos espécimes de vida livre ou aos animais de cativeiro, sendo disponibilizado um pequeno número de animais para realização de pesquisas voltadas para descrição macroscópica.

\section{Conclusão}

A descrição dos constituintes e particularidades do aparelho reprodutor masculino do $P$. yagouaroundi permitiu caracterizar diferenças básicas entre espécies da mesma família, comprovando assim a necessidade de estudos descritivos para cada espécie, pois podem apresentar diferenças entre si, mesmo sendo próximas geneticamente. Os felinos, bem como outros animais, estão em constante adaptação ao ambiente, modificando-se anatomicamente e fisiologicamente para manter sua espécie em condições adversas. Dessa forma, a presença de espículas no $P$. yagouaroundi demonstra uma adaptação morfofuncional comum aos felinos, assim como as demais estruturas identificadas, escroto, pênis, testículos, epidídimos, ductos deferentes e as glândulas genitais acessórias, semelhantes ao descrito para o gato doméstico, exceto por não ter sido identificado as glândulas bulbouretrais na espécie estudada.

\section{Referências Bibliográficas}

Almeida, L. B., Queirolo, D., Beisiegel, B. M. \& Oliveira, T. G. 2013. Avaliaçao do estado de conservaçao do gato-mourisco Puma yagouaroundi (E. Geoffroy Saint-Hilaire, 1083) no Brasil. Biodiversidade Brasileira, 3, 99-106.

Baptista, R. I. A. A., Silva, J. M. \& Oliveira, M. A. B. 2016. Comportamento do gato-mourisco (Puma yagouaroundi, É. Geoffroy, 1803) em cativeiro. IX Jornaada de ensino, pesquisa e extensão - JEPEX. Recife, Pernambuco.

Bressan, P. M., Kierulff, M. C. M. \& Sugieda, A. M. 2009. Fauna ameaçada de extinção no Estado de São Paulo, São Paulo, São Paulo.
Carneiro, R. M., Branco, É., Pinheiro, L. L., Martins, D. M., Santa, B. S., Araújo, E. B., Souza, A. C. B., Pereira, L. C. \& Lima, A. R. 2010. Descrição morfológica do sistema reprodutor masculino de jaguatirica (Leopardus pardalis). Biotemas, 23, 83-89.

Cubas, Z. S., Silva, J. C. R. \& Dias, J. L. C. 2014. Tratado de animais selvagens-medicina veterinária. Editora Roca.

Dyce, K. M., Wensing, C. J. G. \& Sack, W. O. 2004. Tratado de anatomia veterinária. Elsevier Brasil, São Paulo.

Harcourt, A. H. \& Gardiner, J. 1994. Sexual selection and genital anatomy of male primates. Biological Sciences, 255, 47-53.

Jayaprakash, D., Patil, S. B., Kumar, M. N., Majumdar, K. C. \& Shivaji, S. 2001. Semen characteristics of the captive Indian leopard, Panthera pardus. Journal of Andrology, 22, 25-33.

Maffei, L., Noss, A. \& Fiorello, C. 2007. The jaguarundi (Puma yagouaroundi) in the kaaiya del gran Chaco National Park, Santa Cruz, Bolivia. Mastozoología Neotropical, 14, 263266.

Mansfield, K. G. \& Land, E. D. 2002. Cryptorchidism in Florida panthers: prevalence, features, and influence of genetic restoration. Journal of Wildlife Diseases, 38, 693-698.

Marques, A. A. B., Fontana, C. S., Vélez, E., Bencke, G. A., Schneider, M. \& Reis, R. E. 2002. Lista de referência da fauna ameaçada de extinção no Rio Grande do Sul.

Moro-Rios, R. F., Silva-Pereira, J. E., Silva, P. W., Moura-Brito, M. \& Nogarolli, D. 2008. Manual de rastros da fauna paranaense. In: Paraná, I. A. d. (ed.). Curitiba.

Queiroz, V. S. 2003. Estudo do efeito das condições de manipulação do sêmen de jaguatiricas (Leopardus pardalis, Linnaeus, 1758) sobre a capacitação e a integridade morfológica e funcional dos espermatozóides. Universidade de São Paulo, São Paulo, Brasil.

\section{Article History:}

Received 31 March 2017

Accepted 1 June 2017

Available on line 20 June 2017

License information: This is an open-access article distributed under the terms of the Creative Commons Attribution License 4.0, which permits unrestricted use, distribution, and reproduction in any medium, provided the original work is properly cited. 